\title{
Anisotropic Exchange Interaction between $p$-Shell Electron and $s$-Shell Hole in CdTe/ZnTe Quantum Dots
}

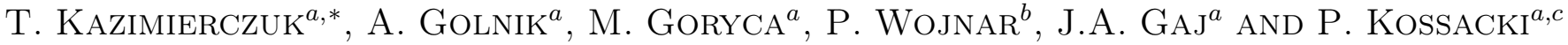 \\ ${ }^{a}$ Institute of Experimental Physics, University of Warsaw, Hoża 69, 00-681 Warsaw, Poland \\ ${ }^{b}$ Institute of Physics, Polish Academy of Sciences, al. Lotników 32/46, 02-668 Warsaw, Poland \\ ${ }^{c}$ Grenoble High Magnetic Field Laboratory, CNRS, BP 16638042 Grenoble Cedex 9, France
}

\begin{abstract}
We study electron-hole exchange interaction in a single CdTe/ZnTe quantum dot by polarization-resolved photoluminescence measurements. We focus on recombination of excitonic states involving $p$-shell electrons: $\mathrm{X}^{2-}$ and $\mathrm{XX}^{-}$. Recombination lines of $\mathrm{X}^{2-}$ and $\mathrm{XX}^{-}$states exhibit fine structure, which can be consistently explained within a model with four parameters $\delta_{i}^{\alpha \beta}$ representing strength of iso- and anisotropic parts of interaction between $s$-hole and $s$ - or $p$-electron.
\end{abstract}

PACS numbers: 78.67.Hc, 78.55.Et

\section{Introduction}

In-plane anisotropy of semiconductor quantum dot (QD) attracted wide attention as an obstacle on the way to generation of entangled photon pairs. Accordingly, the central point of most of the studies in the area focused on the splitting of the neutral exciton (X) and biexciton (XX) transitions which probes the anisotropic part of exchange interaction between electron and holes in the $s$-shell. In the presented work, we analyze the anisotropic effects related to charged biexciton $\left(\mathrm{XX}^{-}\right)$ and doubly charged exciton $\left(\mathrm{X}^{2-}\right)$ and determine the parameters describing exchange interaction between $p$-shell electron and $s$-shell hole in a single CdTe/ZnTe QD.

\section{Experimental}

The experiments were carried out on a sample containing self-assembled CdTe/ZnTe QDs grown by the tellurium desorption method [1].

The experiments were performed in a microphotoluminescence $(\mu \mathrm{PL})$ setup. The sample was mounted on a microscope objective [2] and placed inside a cryostat at $T=1.6 \mathrm{~K}$. QDs were excited non-resonantly by frequency doubled femtosecond Ti:sapphire laser. The light emitted by the QDs was collected by a set of lenses, passed through linear polarizer and was resolved in a monochromator with a CCD camera. A $\lambda / 2$ wave-plate placed in front of the polarizer was used to control the polarization orientation of the recorded light.

A spectrally separated set of lines related to emission from a single $\mathrm{QD}$ was observed in the $\mu \mathrm{PL}$ spectrum (Fig. 1). Identification of optical transitions in the PL spectrum was based on relative energy positions and

* corresponding author; e-mail: Tomasz.Kazimierczuk@fuw.edu.pl polarization properties as well as on photon correlation measurements performed on individual QD PL lines. The latter experiments were performed in a Hanbury-Brown and Twiss setup with avalanche photodiodes. Its detailed description is given in [3]. Systematic photon correlation experiments allowed us to attribute charge states to all the transitions and establish the order of transitions in recombination cascades. The obtained data was used, inter alia, to identify lines related to recombination of neutral exciton $(\mathrm{X})$, biexciton $(\mathrm{XX})$, charged excitons $\left(\mathrm{X}^{+}, \mathrm{X}^{-}\right.$, and $\left.\mathrm{X}^{2-}\right)$, and charged biexciton $\left(\mathrm{XX}^{-}\right)$.

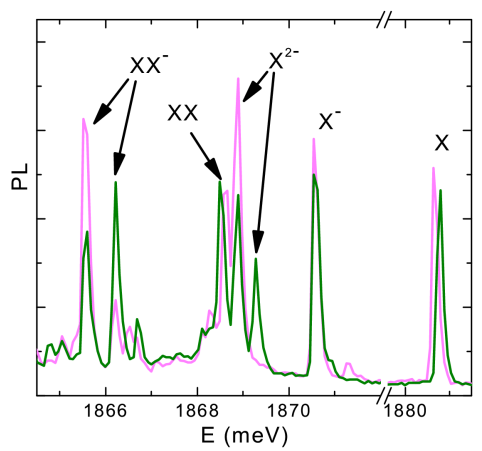

Fig. 1. PL spectrum of a single quantum dot in linear polarizations along the principal QD axes.

The PL spectrum of the QD depended on the orientation of detected polarization which was recognized as a fingerprint of the in-plane anisotropy. Two perpendicular orientations characterized by extremal PL changes (e.g. minimal or maximal spectral line intensity) were identified as principal axes of the QD. The determination of the principal axes was necessary as in II-VI materials orientation of the in-plane anisotropy is not related to the crystallographic axes [4]. 


\section{Results}

The example PL spectrum of a typical QD in linear polarizations along the principal axes of the QD is presented in Fig. 1. Several studied QDs exhibited similar emission patterns. As expected, no splitting of $\mathrm{X}^{-}$was detected while excitonic and biexcitonic transitions were split into pairs of linearly polarized lines. The splittings of $\mathrm{X}$ and $\mathrm{XX}$ lines have the same value but opposite sign. For this quantum dot it was equal to $50 \mu \mathrm{eV}$.

A more complex structure was observed for lines related to recombination of $\mathrm{X}^{2-}$ and $\mathrm{XX}^{-}$states. Both transitions were split into doublets linearly polarized along the same two principal axes. However, the degree of polarization of split components varied from $25 \%$ to $100 \%$. In order to interpret the observed structure, we used a Hamiltonian of anisotropic interaction between electrons (e) and heavy holes (h) [5]. We neglect configuration mixing for the simplicity of the model. We decompose the Hamiltonian of the $n$ carrier complex into two-particle components $H_{\mathrm{e}-\mathrm{h}}$ summed over each e-h pair. We assume that $H_{\mathrm{e}-\mathrm{h}}$ has the same form as in the neutral exciton with parameters $\delta_{0}, \delta_{1}, \delta_{2}$ dependent only on the involved shells (denoted by superscript $x y$ where $x$ and $y$ are the shells of the hole and the electron, respectively). In case of the neutral exciton the isotropic part $\delta_{0}$ is much larger than anisotropic part $\delta_{1}[6]$ which in turn is much larger than $\delta_{2}$. We arbitrarily fix $\delta_{2}^{s s}$ and $\delta_{2}^{s p}$ to a negligible value of $1 \mu \mathrm{eV}$ in our calculations. We also assumed that the e-e exchange interaction is much stronger than the $\mathrm{e}-\mathrm{h}$ exchange interaction responsible for QD fine structure. Thus, we describe the exchange interaction in our system by 4 free parameters: $\delta_{0}^{s s}, \delta_{1}^{s s}, \delta_{0}^{s p}$ and $\delta_{1}^{s p}$.

The energy level structures revealed by diagonalization of the Hamiltonian are presented in Fig. 2. The splitting of $\mathrm{X}$ and $\mathrm{XX}$ lines yields directly the value of anisotropic $s s$ interaction $\delta_{1}^{s s}$ (Fig. 2a).

In the case of $\mathrm{X}^{2-}$ recombination, the structure of the initial state $(3 \mathrm{e} 1 \mathrm{~h})$ is governed by exchange interaction between a single unpaired electron and the hole while there is no $\mathrm{e}-\mathrm{h}$ exchange interaction in the final state (2e). Therefore, the expected splittings follow a pattern of neutral exciton $\mathrm{X}$ but are parameterized by $\delta_{i}^{s p}$ instead of $\delta_{i}^{s s}$. The calculated selection rules are presented in Fig. 2a,c. In the experiment, the $\mathrm{X}^{2-}$ transition was split into a pair of lines of clearly different intensities. The stronger line exhibited linear polarization degree of 0.25 while the weaker one was fully polarized along perpendicular direction. This pattern is well reproduced by the model if we assume $\delta_{1}^{s p} \approx 2 \delta_{0}^{s p}$ (Fig. $2 \mathrm{~d}$ ).

The structure of $\mathrm{XX}^{-}$transition originates from the splittings in its final state of $2 \mathrm{e} 1 \mathrm{~h}$. No interaction is present in the initial state due to filled $s$-shell of holes. The final state consists of three carriers: a hole, an $s$-shell electron and a $p$-shell electron interacting with each other. Following the fact that e-e exchange interaction is much stronger than $\mathrm{e}-\mathrm{h}$ exchange interaction [7], we reduce the considered space to configurations
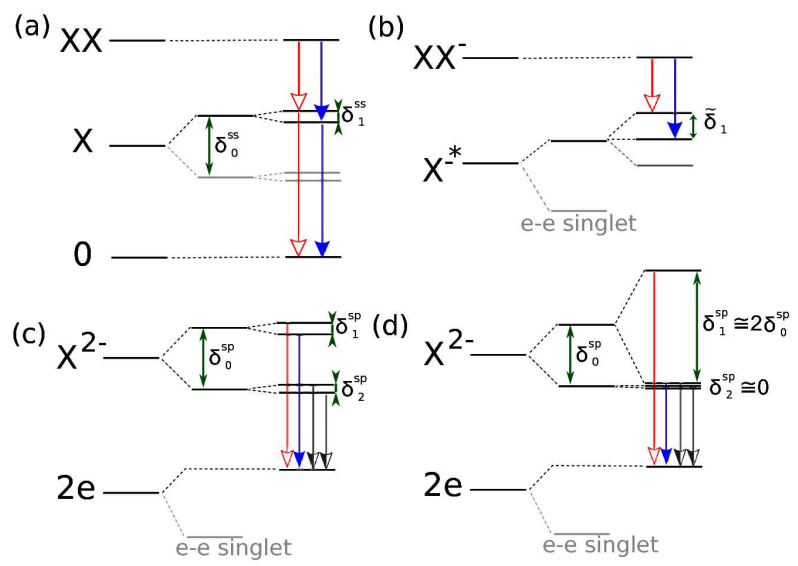

Fig. 2. Theoretical structures (not in scale) of various transitions in QD: (a) neutral exciton and biexciton, (b) charged biexciton, (c) doubly charged exciton. Scheme (d) presents structure of doubly charged exciton in case of studied QDs. Full and empty arrowheads denote orientation of linear polarization of the transitions. Half-full arrowheads represent unpolarized transitions.

with electrons forming a triplet. A diagonalization of the Hamiltonian reveals splitting into 3 levels: one dark and two bright ones, separated by $\widetilde{\delta_{0}}=\delta_{0}^{s s}+\delta_{0}^{s p}$. The anisotropic part $\widetilde{\delta_{1}}=\delta_{1}^{s s}+\delta_{1}^{s p}$ leads to partial linear polarization of both visible transitions. This simulated scheme reproduces our experimental observations with parameters: $\widetilde{\delta_{0}}=1.3 \mathrm{meV}$ and $\widetilde{\delta_{1}} / \widetilde{\delta_{0}}=0.3$. These values are consistent with the ones obtained previously within the experimental accuracy of our setup.

\section{Conclusion}

Summarizing, the observed fine structure of $\mathrm{X}^{2-}$ and $\mathrm{XX}^{-}$states can be consistently explained within a model with four parameters $\delta_{i}^{\alpha \beta}$ representing strength of isoand anisotropic parts of interaction between $s$-hole and $s$ - or $p$-electron. The determined values: $\delta_{0}^{s s}=1.1 \mathrm{meV}$, $\delta_{1}^{s s}=0.05 \mathrm{meV}, \delta_{0}^{s p}=0.20 \mathrm{meV}, \delta_{1}^{s p}=0.40 \mathrm{meV}$.

These results follow a pattern observed previously for CdSe QDs [7]. The absolute values of $\delta_{i}^{\alpha \beta}$ are nearly $50 \%$ lower than those reported for CdSe dots in Ref. [7].

\section{Acknowledgments}

This work was partially supported by the Polish Ministry of Science and Higher Education as research grants in years 2006-2010 and by European Project No. MTKD-CT-2005-029671. One of us (P.K.) acknowledges the support from European Project No. FP7/2007-2013-221515 (MOCNA). 


\section{References}

[1] P. Wojnar, J. Suffczyński, K. Kowalik, A. Golnik, M. Aleszkiewicz, G. Karczewski, J. Kossut, Nanotechnology 19, 235403 (2008).

[2] J. Jasny, J. Sepiol, T. Irngartinger, M. Traber, A. Renn, U.P. Wild, Rev. Sci. Instrum. 67, 1425 (1996).

[3] J. Suffczyński, T. Kazimierczuk, M. Goryca, B. Piechal, A. Trajnerowicz, K. Kowalik, P. Kossacki, A. Golnik, K.P. Korona, M. Nawrocki, J.A. Gaj, Phys. Rev. B 74, 085319 (2006).

[4] T. Kazimierczuk, J. Suffczyński, A. Golnik, J.A. Gaj, P. Wojnar, P. Kossacki, J. Korean Phys. Soc. 53, 154 (2008).
[5] D. Gammon, E.S. Snow, B.V. Shanabrook, D.S. Katzer, D. Park, Science 273, 87 (1996).

[6] M. Bayer, G. Ortner, O. Stern, A. Kuther, A.A. Gorbunov, A. Forchel, P. Hawrylak, S. Fafard, K. Hinzer, T.L. Reinecke, S.N. Walck, J.P. Reithmaier, F. Klopf, F. Schäfer, Phys. Rev. B 65, 195315 (2002).

[7] I.A. Akimov, K.V. Kavokin, A. Hundt1, F. Hennebergerl, Phys. Rev. B 71, 075326 (2005). 